\title{
Síntese de Diagramas de Irradiação de Redes de Antenas Utilizando o Método de Colônia de Morcegos
}

\author{
Ricardo S. Senandes, Marcos V. T. Heckler e Edson R. Schlosser
}

\begin{abstract}
Resumo-Este trabalho apresenta uma nova abordagem para a síntese de diagramas de irradiação utilizando o algoritmo bioinspirado em colônia de morcegos. Esse algoritmo é apresentado e aplicado à síntese de diagramas de irradiação para redes lineares de antenas, utilizando antenas isotrópicas e em microfita retangulares. Como principais contribuições, destacam-se a verificação da eficácia do método para síntese de redes lineares e a necessidade de consideração de diagramas de irradiação realísticos para projeto de sistemas de comunicação sem fio.
\end{abstract}

Palavras-Chave-Antena de microfita, redes de antenas, métodos de otimização, síntese de diagramas de irradiação, colônia de morcegos.

Abstract-This work presents a new approach for the synthesis of radiation patterns using the bio-inspired algorithm based on bat colonies. This algorithm is presented and applied to the synthesis of linear antenna arrays using isotropic antennas and rectangular microstrip antennas. The main contributions are the demonstration of the efficiency of the method and the need to consider realistic patterns for the single elements to design antennas for wireless communication systems.

Keywords-Microstrip antenna, antenna arrays, optimization methods, synthesis of radiation patterns, bat colony.

\section{INTRODUÇÃO}

Métodos de síntese de diagramas de irradiação são fundamentais no projeto de redes de antenas impressas que necessitam de apontamento do feixe principal e controle dos lóbulos secundários, modelamento do feixe, inserção de nulos em determinadas direções, etc [1], visto que as amplitudes e as fases dos coeficientes de excitação dos elementos que compõem a estrutura não podem ser calculadas analiticamente de forma precisa. Diversos métodos podem ser encontrados na literatura para as respectivas aplicações, podendo-se citar o Algoritmo Genético (GA), o Método de Enxame de Partículas (PSO), o Método de Colônia de Formigas (ACO), o Método de Colônia de Vaga-lumes (FA), entre outros.

O GA consiste em gerar inicialmente um conjunto de possíveis soluções, compostas de um conjunto particular de amplitudes e fases para excitação das antenas, e executa

Ricardo Souza Senandes, Programa de Pós-Graduação em Engenharia Elétrica, Universidade Federal do Pampa, Alegrete-RS, e-mail: ricardosenandes.aluno@unipampa.edu.br; Marcos Vinício Thomas Heckler, Programa de Pós-Graduação em Engenharia Elétrica, Universidade Federal do Pampa, Alegrete-RS, e-mail: marcosheckler@unipampa.edu.br; Edson Rodrigo Schlosser, Programa de Pós-Graduação em Engenharia Elétrica, Universidade Federal do Pampa, Alegrete-RS, e-mail: edsonschlosser@unipampa.edu.br. O presente trabalho foi realizado com apoio da Coordenação de Aperfeiçoamento de Pessoal de Nível Superior - Brasil (CAPES) - Código de Financiamento 001 . algumas operações ao longo do processo iterativo, tais como cruzamento e mutação. Após o processo de otimização, obtémse uma solução ótima que contém a combinação de amplitudes e fases obtidas durante o processo evolutivo. Os coeficientes de excitação determinados correspondem aos valores de diferentes soluções do conjunto inicial e/ou da mutação. Por este motivo, o GA apresenta dificuldade para convergir para um ótimo local. Já o PSO realiza a mudança das amplitudes e fases a cada iteração para todas as soluções, ou seja, as variáveis de síntese são alteradas dinamicamente e podem assumir qualquer valor dentro do espaço de busca. A forma de interação entre as soluções possibilita ao método buscas local e global simultaneamente, impactando na rápida convergência para uma ótima solução. As técnicas GA e PSO foram aplicadas na otimização de antenas em [2], [3], em que os autores apresentam as vantagens no desempenho do PSO em comparação ao GA. Em [4], o projeto de uma rede de monopolos com distribuição uniforme de potência no plano azimutal e minimização dos lóbulos laterais é proposto para ser embarcado em um Veículo Aéreo Não-Tripulado (VANT), em que os coeficientes de excitação dos monopolos foram determinados a partir do PSO.

O ACO é uma técnica que consiste em discretizar os intervalos de busca das amplitudes e fases de alimentação, conforme discutido em [5], [6]. Assim, a solução converge apenas para os valores discretos (caminhos) definidos no início da análise. A partir da atratividade entre as formigas, dadas pelo feromônio, o problema é otimizado e os valores discretos são escolhidos. Esse método é interessante em aplicações que envolvem grandezas discretizadas, como, por exemplo, defasadores e amplificadores de ganho variável digitais, pois estes dispositivos são controlados digitalmente através de um número limitado de bits.

O FA é um método de otimização baseado na bioluminescência, em que os vaga-lumes contendo as amplitudes e fases são atraídos em direção a uma ótima solução. A atratividade entre insetos é reduzida em função da distância no espaço de busca e pela taxa de decaimento da luz. Essa técnica foi aplicada em [7] para se determinar as distâncias entre elementos de uma rede linear não-uniforme e em [8] para definir os coeficientes de excitação de redes planares de antenas instaladas em um nanossatélite.

Neste trabalho o método de colônia de morcegos (do inglês, bat algorithm - BA) é implementado e investigado em problemas que envolvem a síntese de diagramas de irradiação. A necessidade desse estudo está relacionada à atualização dos 
algoritmos já aplicados ao problema de síntese por novos que apresentem maior robustez.

$\mathrm{O}$ artigo está estruturado da seguinte maneira: na seção II é descrito o algoritmo BA, enquanto que nas seções III e IV são apresentadas análises de redes lineares de antenas compostas por antenas isotrópicas e de microfita. Finalmente, as principais conclusões do trabalho são apresentadas na seção V.

\section{Algoritmo de Colônia de Morcegos (BA)}

O algoritmo bio-inspirado em colônia de morcegos foi desenvolvido por Xin-She Yang em 2010 e baseia-se nas características de ecolocalização desses animais [9]. Os morcegos utilizam o som para detectar presas, obstáculos e fendas no escuro. A ecolocalização ocorre pelo cálculo do tempo decorrido entre a emissão de um pulso e sua respectiva recepção [10]. O algoritmo de colônia de morcegos possui três regras básicas que devem ser consideradas:

1) Todos os morcegos da colônia emitem pulsos para detectar a distância de presas ou de obstáculos;

2) Os morcegos voam de forma aleatória;

3) Os morcegos variam as propriedades dos pulsos conforme sua estratégia de busca.

A Figura 1 ilustra uma colônia de morcegos emitindo e recebendo pulsos sonoros durante seu voo.

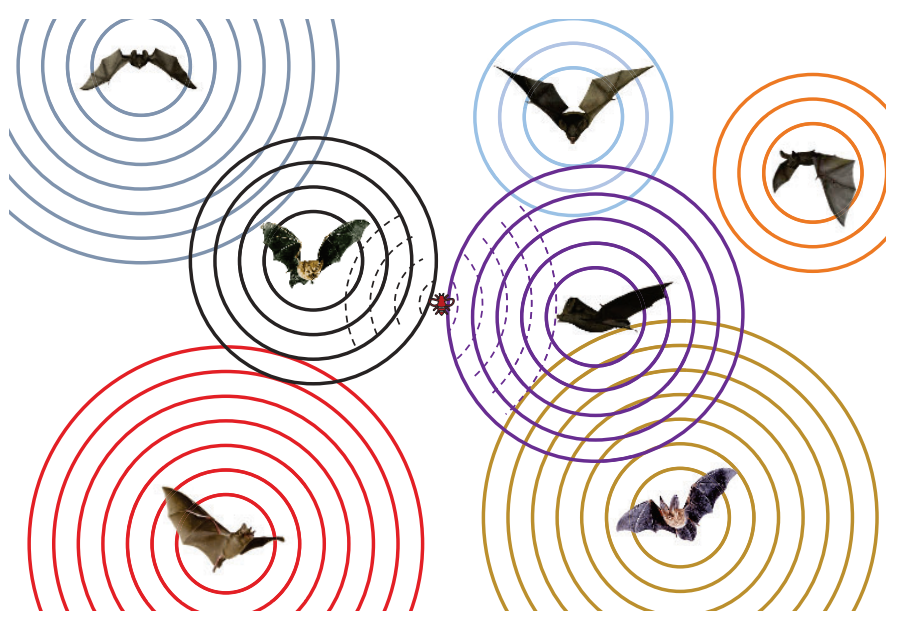

Fig. 1. Colônia de morcegos emitindo e recebendo pulsos durante o voo em busca de alimento ou para localização.

O tamanho da colônia varia conforme a aplicação do algoritmo e o tamanho do espaço de busca. Tipicamente, utilizamse entre 20 e 40 morcegos para problemas com poucas dimensões. Cada morcego tem dimensão $d$, que corresponde ao número de variáveis a serem otimizadas [9], [10]. Portanto, a colônia é formada por $n$ morcegos correspondentes a vetores multidimensionais de tamanho $d$ e que representam possíveis soluções para o problema. Neste trabalho, cada morcego $i$ da colônia é representado matematicamente por

$$
x_{i}=\left[\begin{array}{lllllll}
a_{1} & \delta_{1} & a_{2} & \delta_{2} & \ldots & a_{d} & \delta_{d}
\end{array}\right],
$$

onde $a_{n}$ e $\delta_{n}$ representam as amplitudes e fases dos coeficientes de excitação do $n$-ésimo elemento de uma rede de antenas, respectivamente.

\section{A. Movimentação dos morcegos}

A cada iteração $t$, a posição $x_{i}^{t}$ e a velocidade $v_{i}^{t}$ do $i$-ésimo morcego da colônia são atualizadas por

$$
\begin{gathered}
f_{i}=f_{\min }+\left(f_{\max }-f_{\min }\right) \beta \\
v_{i}^{t+1}=v_{i}^{t}+\left(x_{i}^{t}-x_{\text {best }}\right) f_{i} \\
x_{i}^{t+1}=x_{i}^{t}+v_{i}^{t+1}
\end{gathered}
$$

sendo que $f_{i}$ é a frequência de emissão do pulso sonoro, $f_{\min }$ e $f_{\max }$ são as frequências mínima e máxima de pulso sonoro, respectivamente, que a colônia pode assumir [9], [10]. O termo $\beta$ é um fator que insere aleatoriedade ao algoritmo, estando dentro do intervalo [0,1] e com distribuição normal. A grandeza $v_{i}^{t}$ é a velocidade de cada morcego no instante atual $t$, enquanto que $v_{i}^{t+1}$ corresponde à velocidade atualizada. Finalmente, a variável $x_{\text {best }}$ corresponde à posição do morcego da colônia mais próxima da solução ótima ao problema em análise.

Os delimitadores do espaço de busca do algoritmo são os termos relacionados à frequência $\left(f_{\min }\right.$ e $\left.f_{\max }\right)$ [9], [10]. Portanto, na aplicação do algoritmo para a síntese de redes de antenas, têm-se duas faixas de busca distintas. Usualmente, utilizam-se amplitudes dentro do intervalo $[0,1]$ e fases $\left[0^{\circ}, 360^{\circ}\right]$.

Além das frequências, velocidades e posições, os volumes $A_{i}$ e as taxas de emissão de pulso $r_{i}$ de cada morcego também devem ser atualizadas.

\section{B. Variação de volume e taxa de pulso}

O volume e a taxa de emissão de pulso de cada morcego indicam qual a situação do morcego dentro do espaço de busca e são utilizados para realizar buscas locais. Escolhe-se um morcego aleatoriamente e realiza-se uma busca local por meio de voo aleatório em torno da melhor solução. Essa busca local é realizada por

$$
x_{n o v a}=x_{v e l h a}+\epsilon A^{t},
$$

sendo $\epsilon$ um fator aleatório com distribuição normal dentro do intervalo [-1,1] e $A^{t}$ a média dos volumes de pulsos da colônia [10]. O volume do pulso de cada morcego é atualizado por

$$
A_{i}^{t+1}=\alpha A_{i}^{t},
$$

onde $\alpha$ é um fator de redução do volume que está dentro do intervalo $[0,1]$. Esse fator modela o decaimento do volume conforme os morcegos se aproximam de obstáculos ou presas. Se o volume

$$
A_{i}^{t} \rightarrow 0
$$

então significa que o morcego encontrou uma presa ou obstáculo, de forma que os pulsos não precisem mais ser emitidos. Inversamente ao volume, a taxa de emissão de pulso aumenta, sendo atualizada por

$$
r_{i}^{t+1}=r_{i}^{0}\left(1-e^{-\gamma t}\right),
$$

onde $\gamma>0$ é um fator de crescimento. Se $A_{i}^{t}=0 \operatorname{com} t \rightarrow \infty$, implica que

$$
r_{i}^{t} \rightarrow r_{i}^{0}
$$

e indica que se atingiu o objetivo do processo de otimização [9], [10]. 


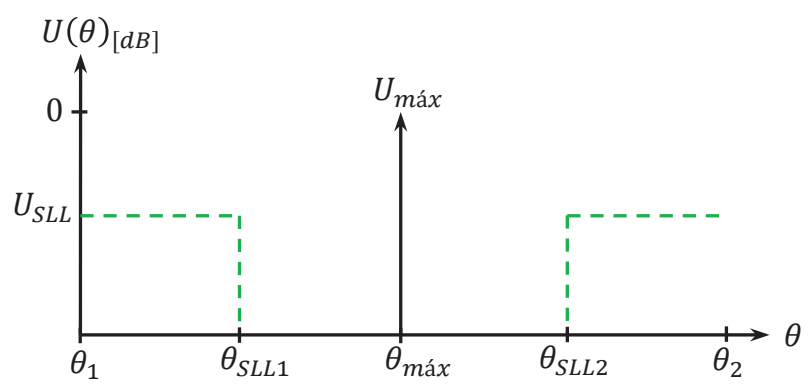

Fig. 2. Máscara utilizada na função objetivo.

\section{Função erro}

Algoritmos são utilizados para otimizar variáveis e, com isso, minimizar algum tipo de função erro. Nesta aplicação, buscam-se otimizar coeficientes de excitação de cada elemento de uma rede de antenas, de modo a sintetizar um diagrama de irradiação desejado. Esse diagrama deve respeitar requisitos de projeto, como, por exemplo, níveis máximos de lóbulos secundários e máxima intensidade de irradiação em uma dada direção de interesse.

Para fazer com que o algoritmo respeite essas exigências, cria-se uma máscara que contém todas as especificações do diagrama de irradiação. Para minimizar a função erro, utilizouse uma máscara que estabelece níveis máximos de lóbulos secundários de intensidade $U_{S L L}$, válidos nas regiões de $\theta_{1}$ a $\theta_{S L L 1}$ e de $\theta_{S L L 2}$ a $\theta_{2}$, e uma direção para apontamento do lóbulo principal para $\theta_{\max }$. Graficamente, esta máscara encontra-se ilustrada na Figura 2.

Para avaliar as potenciais soluções do processo de otimização, define-se, neste trabalho, a função erro como sendo

$$
\operatorname{erro}(\theta)=\sum_{\theta=0^{\circ}}^{180^{\circ}}\left|O(\theta) \operatorname{Peso}(\theta)\left(U_{\text {Mask }}(\theta)-U_{\text {OutB } A}(\theta)\right)\right| \text {, }
$$

onde $U_{\text {Mask }}(\theta)$ é a intensidade de irradiação especificada pela máscara e $U_{O u t B A}(\theta)$ é a intensidade de irradiação calculada a partir dos coeficientes de excitação obtidos pelo BA, sendo ambas normalizadas. Em (10), ambos os termos $U_{\text {Mask }}(\theta)$ e $U_{\text {OutBA }}(\theta)$ encontram-se em decibéis e normalizados de acordo com

$$
U(\theta)_{d B}=10 \log \left(\frac{U(\theta)}{\max (U(\theta))}\right) .
$$

A função $O(\theta)$ é utilizada para computar ou não o erro em um determinado ângulo $\theta$. A função $\operatorname{Peso}(\theta)$ é utilizada para aplicar fatores ponderativos no cálculo do erro, uma vez que os lóbulos laterais têm maior influência no erro total do que o ângulo de máxima intensidade ou apontamento.

\section{REDE LINEAR DE ANTENAS ISOTRÓPICAS}

De acordo com a teoria clássica de antenas, pode-se obter, de forma geral, o campo elétrico total irradiado por uma rede de antenas por

$$
\vec{E}_{T O T A L}(\theta, \phi)=\vec{E}_{E L E M E N T}(\theta, \phi) \cdot A F(\theta, \phi),
$$

onde $\bar{E}_{E L E M E N T}(\theta, \phi)$ é o campo elétrico de um elemento isolado e $A F(\theta, \phi)$ o fator de rede que modela o efeito gerado ao se agrupar antenas. A Figura 3 apresenta uma rede de antenas dispostas ao longo do eixo $x$ positivo, sendo $d_{x}$ a distância entre elementos adjacentes. Neste estudo, o valor adotado foi $d_{x}=\lambda_{0} / 2$.

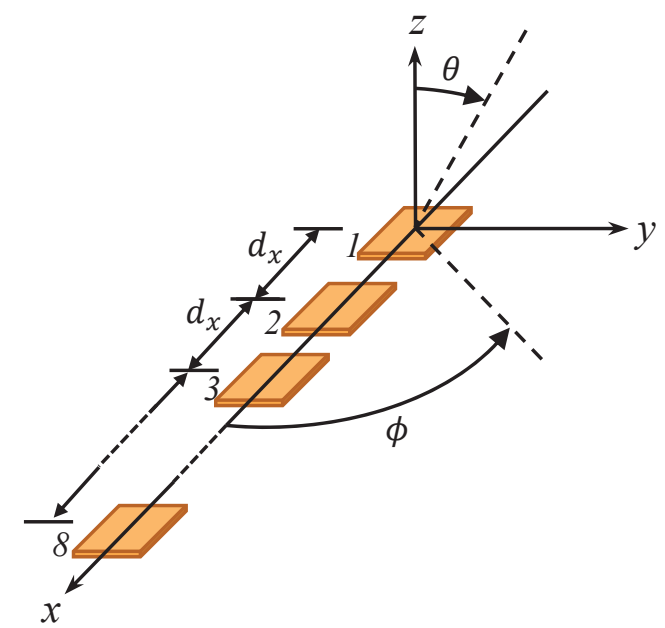

Fig. 3. Rede de antenas disposta ao longo do eixo $x$ e com espaçamento uniforme.

Para verificar a capacidade de otimização do BA, utilizaramse oito antenas isotrópicas, sendo

$$
A F(\theta, \phi)=\sum_{n=1}^{8} a_{n} e^{j\left(k_{0} d_{x}(n-1) \sin \theta \cos \phi+\delta_{n}\right)},
$$

onde $a_{n}$ e $\delta_{n}$ correspondem à amplitude e à fase de excitação do $n$-ésimo elemento da rede. Por fim, o campo elétrico total irradiado por uma rede de antenas isotrópicas é descrito por

$$
E_{\text {TOTAL }}(\theta, \phi)=\sum_{n=1}^{8} a_{n} e^{j\left(k_{0} d_{x}(n-1) \sin \theta \cos \phi+\delta_{n}\right)} .
$$

Para avaliar a capacidade do BA na síntese de diagramas de irradiação, utilizou-se uma colônia com 50 morcegos, com parâmetros $\gamma=0,1$ e $\alpha=0,97$, e um máximo de 50 iterações. Neste trabalho, duas máscaras foram consideradas, ambas com limite de lóbulos secundários de $-20 \mathrm{~dB}$, porém com apontamentos do lóbulo principal para $\theta_{\max }=0^{\circ}$ (broadside) e $\theta_{\max }=40^{\circ}$. A Figura 4 apresenta o diagrama de irradiação sintetizado pelo BA para uma rede linear de 8 antenas isotrópicas com apontamento em broadside, juntamente com a máscara e o diagrama obtido a partir de distribuição uniforme de amplitudes e defasagem progressiva. O diagrama sem otimização apresenta lóbulos secundários maiores que $-15 \mathrm{~dB}$ e um ângulo de primeiro nulo de $30^{\circ}$, não respeitando as especificações da máscara. Já o diagrama sintetizado apresenta níveis de lóbulos secundários menores que $-20 \mathrm{~dB}$, e atende perfeitamente a especificação.

O BA pode ser utilizado também para realizar o apontamento do lóbulo principal em uma direção diferente da broadside. A Figura 5 apresenta o diagrama de irradiação sintetizado pelo BA e também pelo conceito de defasagem progressiva para $\theta_{\max }=40^{\circ}$. Nota-se que o feixe principal tende a alargar em comparação ao primeiro caso abordado. Contudo, o diagrama sintetizado pelo BA ainda respeita o 


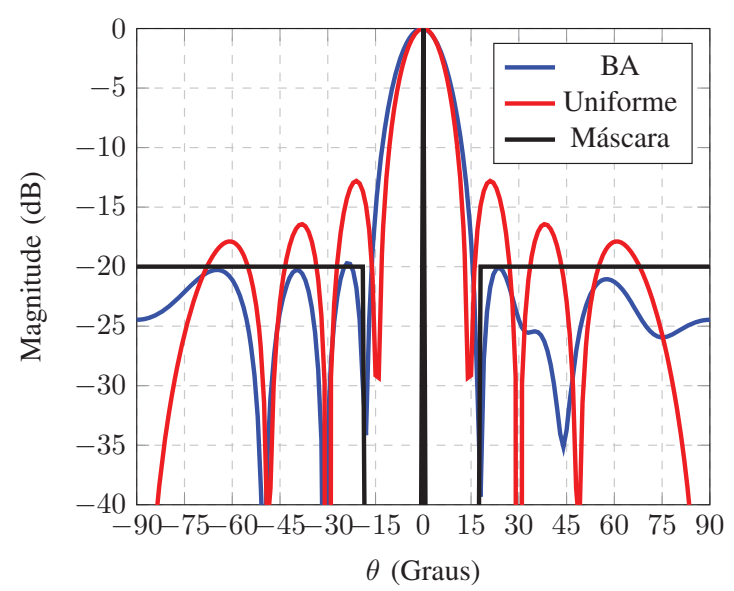

Fig. 4. Diagramas de irradiação de rede de antenas isotrópicas sintetizados pelo BA.

limite máximo imposto aos lóbulos secundários da máscara, enquanto o mesmo não é verificado utilizando defasagem progressiva e amplitudes uniformes.

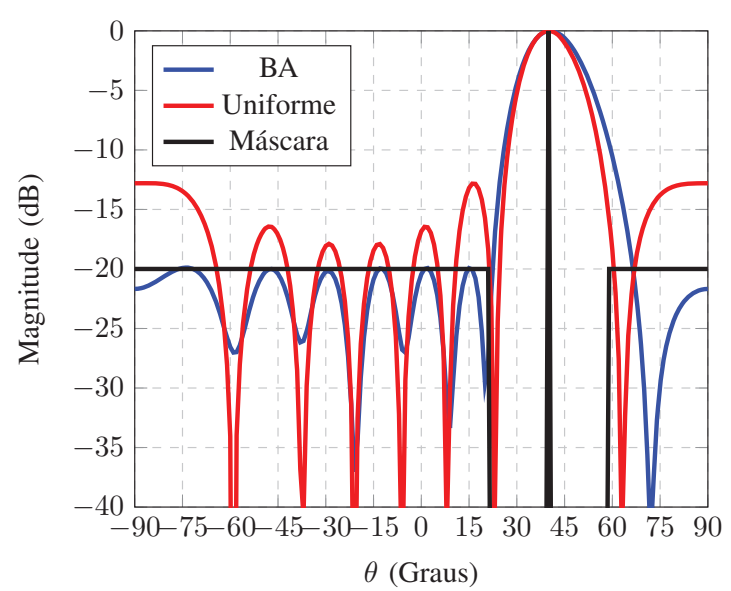

Fig. 5. Diagramas de irradiação com apontamento para $\theta_{\max }=40^{\circ}$ sintetizados pelo BA.

\section{REDE LINEAR DE ANTENAS DE MICROFITA}

Antenas isotrópicas são muito usadas para fins teóricos, uma vez que seu diagrama de irradiação não apresenta variação espacial de intensidade de irradiação. Entretanto, deve-se destacar que, para fins práticos, tais antenas ainda não foram desenvolvidas.

Antenas de microfita são uma classe de irradiadores que se estabeleceu na academia e na indústria, em função de apresentarem inúmeras vantagens, tais como baixo peso, facilidade de construção e baixo custo para produção em larga escala. Assim como todos os demais tipos de irradiadores, as antenas de microfita apresentam características de irradiação diferentes de uma antena isotrópica [1]. A Figura 6 apresenta comparação entre as intensidades de campo elétrico irradiado por antenas isotrópica e impressa. Como pode ser observado, a antena isotrópica tem intensidade de campo igual em todas as direções, enquanto que, para a antena de microfita, a intensidade de irradiação é máxima para $\theta=0^{\circ}$ (direção normal ao plano que contém o elemento irradiador de microfita) e diminui para outros ângulos.

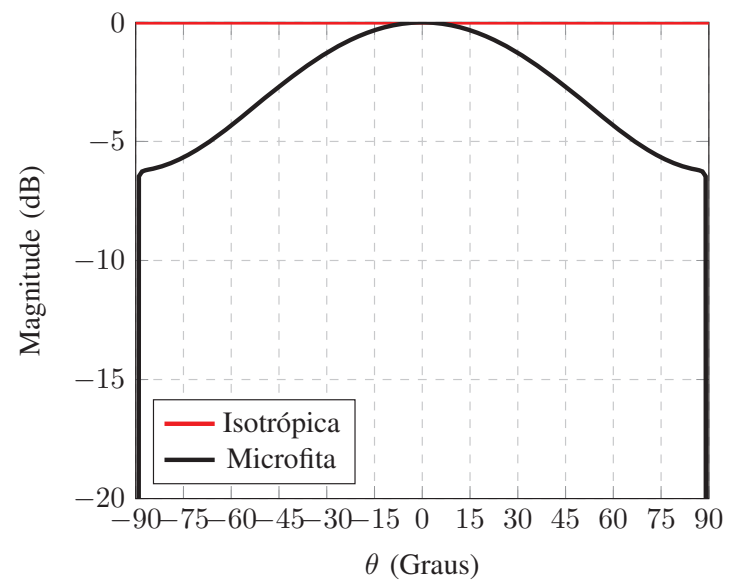

Fig. 6. Intensidade de campo elétrico irradiado por antenas isotropicas e microfita.

Para demonstrar o efeito do diagrama do elemento nas características da rede de antenas, as oito antenas isotrópicas foram substituídas por irradiadores de microfita. Ao se utilizarem os mesmos coeficientes de excitação otimizados com o BA e empregados para gerar os diagramas das Figuras 4 e 5 (curvas azuis), resultam os diagramas apresentados nas Figuras 7 e 8 para $\theta_{\max }=0^{\circ}$ e $\theta_{\max }=40^{\circ}$, respectivamente

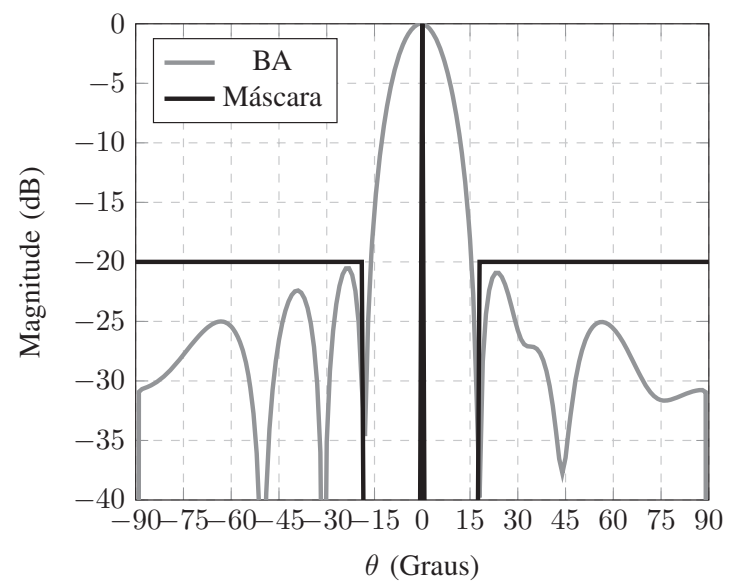

Fig. 7. Aplicação em rede de antenas microstrip dos coeficientes obtidos pelo $\mathrm{BA}$ utilizando antenas isotrópicas para apontamento na direção $\theta_{\max }=0^{\circ}$.

Para o apontamento na direção de broadside, obtiveramse lóbulos secundários ainda abaixo de $-20 \mathrm{~dB}$ de intensidade. Já para o apontamento em $\theta_{\max }=40^{\circ}$, os lóbulos secundários obtidos foram maiores que $-20 \mathrm{~dB}$. Além disso, verifica-se desapontamento do lóbulo principal de cerca de $-2^{\circ}$, desrespeitando a especificação de máxima irradiação da máscara. Dessa forma, fica evidente que a característica de irradiação do tipo de antena empregado para composição da rede afeta diretamente o diagrama de irradiação final. Portanto, o emprego de irradiadores isotrópicos para a síntese de redes de antenas realistas não é adequado. 


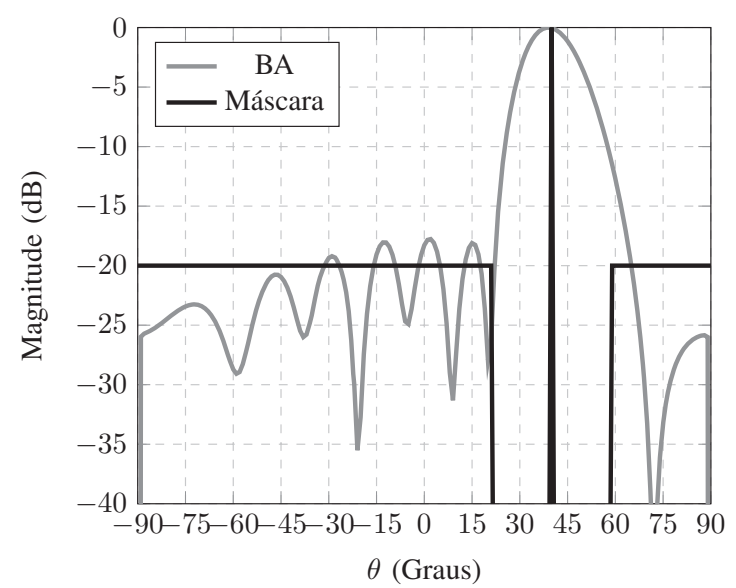

Fig. 8. Aplicação em rede de antenas microstrip dos coeficientes obtidos pelo BA utilizando antenas isotrópicas para apontamento na direção de $\theta=40^{\circ}$.

A partir da verificação acima, o BA foi executado novamente para sintetizar diagramas levando em consideração as características de antenas de microfita. As Figuras 9 e 10 apresentam os diagramas sintetizados para os casos analisados anteriormente, onde, desta vez, observa-se que o diagrama respeitou o limite máximo para os lóbulos secundários, estabelecido na máscara, além do apontamento correto para o lóbulo principal.

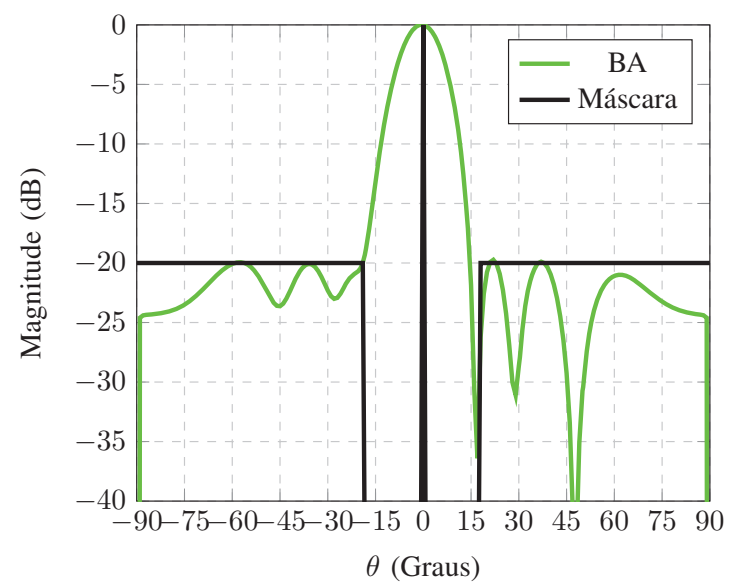

Fig. 9. Diagrama de irradiação sintetizado pelo BA utilizando a intensidade de irradiação de antenas de microfita retangulares com apontamento em broadside.

\section{CONClus Ão}

Neste trabalho, o algoritmo BA foi apresentado e se mostrou um método eficaz para a síntese de diagramas de irradiação de redes de antenas. Apresentaram-se as diferenças obtidas otimizando-se uma rede linear de antenas isotrópicas e de microfita. Ficou evidente que a síntese utilizando antenas isotrópicas não pode ser empregada para projeto de redes de antenas realistas, devendo-se incluir as características de irradiação do tipo de antena a ser usado para composição da rede. O BA respeitou os limites de projeto impostos pela máscara, controlando os níveis de lóbulos secundários e realizando o

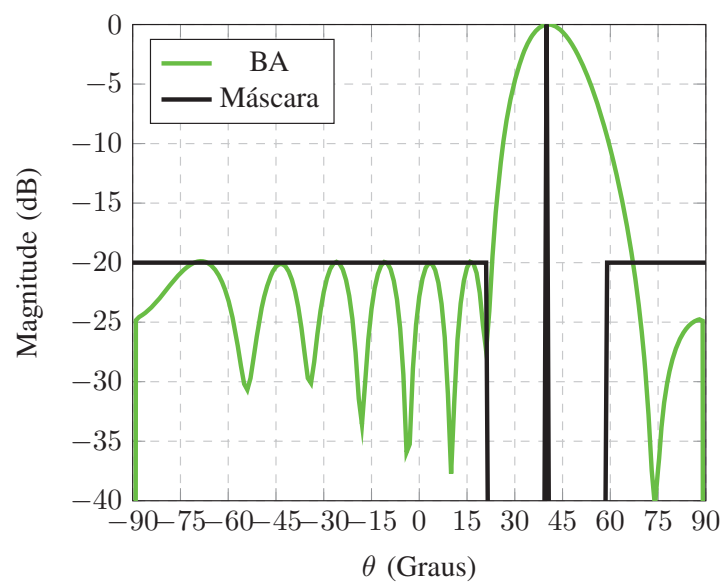

Fig. 10. Diagrama de irradiação sintetizado pelo BA utilizando a intensidade de irradiação de antenas de microfita retangulares com apontamento em $\theta=$ $40^{\circ}$.

apontamento de forma desejada, podendo substituir qualquer algoritmo já proposto para síntese de diagramas.

\section{AgRADECIMENTOS}

O presente trabalho foi realizado com apoio da Coordenação de Aperfeiçoamento de Pessoal de Nível Superior - Brasil (CAPES) - Código de Financiamento 001.

\section{REFERÊNCIAS}

[1] C. A. Balanis, Antenna theory: analysis and design, 3rd ed. John wiley \& sons, 2005

[2] E. R. Schlosser, S. M. Tolfo, M. V. T. Heckler, Particle Swarm Optimization for Antenna Arrays Synthesis. In: SBMO/IEEE MTT-S International Microwave and Optoelectronics Conference, 2015, Porto de Galinhas PE. International Microwave and Optoelectronics Conference, 2015.

[3] W.-B. Wang, Q.-Y. Feng, D. Liu, Synthesis of thinned linear and planar antenna arrays using binary PSO algorithm. Progress In Electromagnetics Research, v. 127, p. 371-387, 2012.

[4] E. Yoshimoto, E. R. Schlosser, M. V. T. Heckler, Optimization of Antenna Arrays Installed on Non-Conductive Unmanned Aerial Vehicle. IET Microwaves Antennas \& Propagation, v. 12, p. 2292-2300, 2018.

[5] E. R. Schlosser, C. Johann, M. V. T. Heckler, Síntese de Redes de Antenas Isotrópicas Aplicando o Método de Colônia de Formigas. In: MOMAG 2016, 2016, Porto Alegre. Momag 2016, 2016.

[6] M. Dorigo, T. Stutzle, Ant Colony Optimization. Cambridge, MA: MIT Press, 2004.

[7] M. A. Zaman, Md. A. Matin, Nonuniformly Spaced Linear Antenna Array Design Using Firefly Algorithm. International Journal of Microwave Science and Technology, v. 2012, 2012.

[8] E. Yoshimoto, M. V. T. Heckler, Optimization of planar antenna arrays using the firefly algorithm. Journal of Microwaves, Optoelectronics and Electromagnetic Applications 18.1 (2019): 126-140.

[9] S. X. Yang, A new metaheuristic bat-inspired algorithm. Springer Berlin Heidelberg, p. 65-74, 2010.

[10] S. X. Yang, A. H. Gandomi, Bat algorithm: a novel approach for global engineering optimization. Engineering computations, 2012 